\section{Ist Fingerknacken schädlich?}

Die Angewohnheit, die Fingergelenke knacken zu lassen, hat wohl keine funktionelle Relevanz, zumindest nicht kurzfristig. Zu diesem Ergebnis kommt eine Studie aus Kalifornien, in der 400 Gelenke unmittelbar vor und nach dem Knacken untersucht wurden. Forscher untersuchten hierzu die Gelenke von insgesamt 40 asymptomatischen Freiwilligen. 30 Teilnehmer waren in der Lage, ihre Metakarpophalangealgelenke knacken zu lassen, die restlichen zehn konnten dies nicht. Wie sich das Knacken allerdings langfristig auf die Gelenke auswirkt, insbesondere im Hinblick auf das Arthroserisiko, bleibt zu untersuchen.

Boutin RD et al. Clin Orthop Relat Res 2017; 475: 1265

\section{Hirnstimulation schaltet Anorexie ab}

Gezielte Stromimpulse in der Area subcallosa können offenbar das Körpergewicht von fast jeder zweiten Anorexiepatientin wieder normalisieren. In einer kleinen Studie mit 16 Patientinnen stieg der BMI deutlich an, auch Depressionen und Zwänge gingen zurück. Vor der tiefen Hirnstimulation (THS) lag der BMI der Frauen zwischen 11,1 und 16,6. Im Laufe von einem Jahr nahm er von im Mittel 13,8 auf 17,3 zu. Ob der Erfolg anhält, muss sich jedoch noch zeigen. Es wären größere randomisiertkontrollierte Studien nötig, um einen Therapieerfolg klar zu belegen, so die Autoren.

Lancet Psychiatry 2017, epub 23.2.17

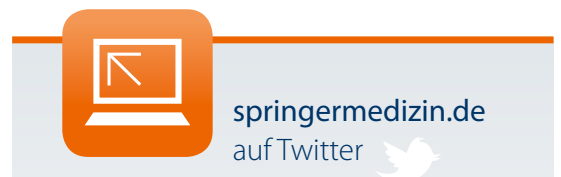

Auch im Frühling heißt es

Lauschen Sie unserem Zwitschern und werden Sie zum Follower.

twitter.com/springermedizin

Dosisabhängige Wirkung

\title{
Mit dem Alkoholverzicht sinkt der Blutdruck
}

Wer mehr als zwei Gläser Alkohol täglich konsumiert, kann seinen Blutdruck durch eine Einschränkung seiner Trinkgewohnheiten senken, so das Ergebnis einer kanadischen Metaanalyse.

Dass Menschen mit übermäßigem Alkoholkonsum ein erhöhtes Hypertonierisiko haben, ist seit langem bekannt. Michael Roerecke vom Institute for Mental Health in Toronto und Kollegen haben sich nun für den umgekehrten Fall interessiert, indem sie die Frage stellten, welche Auswirkungen es auf den Blutdruck hat, wenn die tägliche Zahl der Drinks reduziert wird. Hierzu analysierten die Kanadier die Daten aus 36 relevanten Studien mit 2865 Teilnehmern mit und ohne Hypertonie. Der Blutdruck der Teilnehmer wurde jeweils vor und mindestens sieben Tage nach der Veränderung der Trinkgewohnheiten ermittelt.

\section{Signifikant ab 3 Getränken}

Bei Menschen, die täglich maximal zwei Drinks (12 g reiner Alkohol pro Getränk) zu sich nahmen, zeigten sich keine nennenswerten Effekte des Alkohols auf den Blutdruck. Wer allerdings mehr konsumierte, der profitierte, wenn er sein Trinkpensum verringerte. Bereits ab drei alkoholischen Getränken wirkte sich ein Verzicht in die Nähe der Abstinenz signifikant auf die Blutdruckwerte aus (systolisch $-1,2 \mathrm{mmHg}$, diastolisch $-1,1 \mathrm{mmHg}$ ). (sta)

Roerecke M et al. Lancet Public Health 2017;2: e108-20

\section{Lithogene Veränderungen}

\section{Schwangerschaft begünstigt Nierensteine}

Eine Schwangerschaft bringt Veränderungen mit sich, die eine Nephrolithiasis begünstigen. Während der Schwangerschaft selbst scheint sich dies allerdings weniger auszuwirken als vielmehr danach.

In eine Analyse flossen die Angaben zu Schwangerschaften und Urolithiasis von knapp 3800 Frauen im Alter von maximal 50 Jahren ein. $78 \%$ von ihnen waren mindestens einmal schwanger gewesen. Insgesamt lag die Längsschnittprävalenz für Nephrolithiasis im untersuchten Kollektiv bei $6,4 \%$. Für Frauen, die schwanger gewesen waren, betrug die Quote 7,5\%, für Frauen ohne Schwangerschaften in der Anamnese lag sie bei 3,2\%.Nach Abgleich von Faktoren wie Alter, ethnische Zugehörigkeit, Adipositas, Diabetes, Hormon- einnahme u.a. ergab sich eine nach der Zahl der Schwangerschaften abgestufte Risikoverteilung. So betrug die adjustierte Nephrolithiasisprävalenz für Nulligravidae $5,2 \%$ und steigerte sich auf $12,4 \%$ bei Frauen mit drei oder mehr Schwangerschaften. Wurden Frauen ohne Schwangerschaften als Referenzgruppe betrachtet, erhöhte eine Schwangerschaft das Nierensteinrisiko um rund $40 \%$. Zwei Schwangerschaften verdoppelten es, bei drei oder mehr lag es rund zweieinhalbfach höher. Die Autoren fordern weitere Untersuchungen, um modifizierbare Risikofaktoren von Schwangeren zu identifizieren. Damit könne die durch Nephrolithiasis verursachte Krankheitslast für Frauen womöglich gesenkt werden. 